\title{
Residual fluctuations in the microwave background at large angular scales: Revision of the Sachs-Wolfe effect
}

\author{
Joan Josep Ferrando, Juan Antonio Morales, and Miquel Portilla \\ Departament de Física Tèrica, Universitat de Valencia, 46100 Burjassot, Valencia, Spain
}

(Received 2 April 1992)

\begin{abstract}
In this paper we revise the Sachs-Wolfe (SW) computation of large-scale anisotropies of the microwave background temperature, taking into account the properties of the metrics admitting an isotropic distribution of collisionless photons. We show that the metric used by SW belongs to the aforementioned class, and conclude that the microwave background (once the dipolar anisotropy has been subtracted) should now be isotropic at large angular scales, provided that it was isotropic on the last scattering surface and assuming that the growing mode of a pressureless Einstein-de Sitter perturbation is a good description of the metric.
\end{abstract}

PACS number(s): $98.70 . \mathrm{Vc}, 98.80 . \mathrm{Hw}$

\section{INTRODUCTION}

Up to date measurements of the cosmic microwave background (CMB) temperature show only dipolar anisotropy; technical refinements are providing smaller upper limits for temperature anisotropies at different angular scales. In the framework of big bang models this highly isotropic CMB is interpreted as a relic of a hot past age of the Universe, when matter and radiation were strongly coupled. If the Universe was then exactly homogeneous and isotropic (a Robertson-Walker universe), the CMB could have entered the recombination era as highly isotropic and with a blackbody spectrum [1]. However, to understand the origin of the structures observed at present, one needs to assume that in the early Universe inhomogeneities existed which were much bigger than statistical fluctuations. The problem is how these irregularities are imprinted in the CMB, and if these predictions are compatible with the upper limits observed. The physical processes involved in this problem depend on the angular scale of the observation. At large scales the physics becomes simpler. The horizon at redshift $z$ subtends now an angle $\theta_{h} \approx 1 / \sqrt{1+z}$ (we are considering an Einstein-de Sitter background) and, assuming recombination occurs at $z \approx 1000$, one gets $\theta_{h} \approx 1.8^{\circ}$. Therefore angular scales greater than $\theta_{h}$ correspond to structures that are bigger than the horizon at decoupling time, and so scattering with electrons can be neglected. On the other hand, reionization may occur later in the process of galaxy formation. Using general arguments one can justify [2] that if this process starts at $z \approx 30$, scattering with electrons may erase primordial anisotropies at angular scales smaller than $\theta_{s} \approx 6^{\circ}$, introducing new ones linked to the peculiar motion of the plasma. According to these considerations, anisotropies at angular scales bigger than $\theta_{s}$ are unaffected by electron scattering. For this reason one expects that measurements of CMB anisotropy at these angular scales (bigger than $\theta_{s}$ ) will probe the distribution of matter on scales larger than the horizon at decoupling time. In this case, CMB anisotropies are ex- pected because of gravitational potential fluctuations. This effect was first stated by Sachs and Wolfe (SW) [3] on considering the growing mode of a perturbed pressureless Einstein-de Sitter universe $(k=0)$, and assuming that radiation, as measured by an observer comoving with matter, was isotropic on the last scattering surface $\Sigma_{d}$. This was defined in physical terms as a surface of constant temperature $T=T_{d}$, and expressed, in the coordinates used by them, as a surface of constant time $\bar{\eta}=\bar{\eta}_{d}$.

The aim of this paper is to revise this procedure. So, in Sec. II, we recall well-known results concerning the existence of isotropic solutions to the Liouville equation for massless particles in an inhomogeneous space-time. In Sec. III we show that the metric used in the SW paper is not compatible with the assumption of an isotropic distribution of collisionless photons with uniform temperature on a hypersurface $\bar{\eta}=$ const in comoving time. The conclusion drawn is that radiation will remain isotropic if it was so at the recombination epoch.

\section{SPACE-TIMES ADMITTING ISOTROPIC RADIATION}

Radiation can be described as a gas of photons with a distribution function $f(x, p), p^{2}=0$. If scattering with electrons can be neglected (or if radiation is in equilibrium with matter) the distribution function must satisfy the Liouville equation

$$
f(x(\lambda), p(\lambda))=\text { const },
$$

$x(\lambda)$ being any null geodesic and $p(\lambda)=d x / d \lambda$. As one is interested in the temperature measured by a given observer, it is preferable to write the Liouville equation in terms of specific intensity. Let us consider a unit timelike vector field $n$, the frequency $v$ and the specific intensity $I_{v}$ measured by observer $n$ are given by $v=-n \cdot p$ and $I_{v}=h^{4} v^{3} f$, respectively. From the Liouville equation one gets $I_{v} / v^{3}=$ const along any light ray, and denoting any 
two events connected by a null geodesic as $x_{e}$ and $x_{0}$, one has

$$
I_{v 0}=\frac{I_{v e}}{(1+z)^{3}}
$$

where $z$ is the redshift corresponding to the observers $n_{e}$ and $n_{0}$ at $x_{e}$ and $x_{0}$, respectively. Expressing $I_{v}$ in terms of an effective temperature $T$, one gets the relation

$$
T_{0}=\frac{T_{e}}{1+z}
$$

If the radiation is isotropic with respect to an observer $n$, the distribution function depends on $p$ through the frequency measured by $n, f(x, p)=j(x, v)$. Tauber and Weinberg [4] and Ehlers, Geren, and Sachs [5] have studied the conditions imposed on the metric and the vector field $n$ so that the solution to the Liouville equation is isotropic. We have recently treated the problem of getting inhomogeneous solutions to Einstein's equations admitting isotropic radiation [6]. The results concerning an isotropic distribution function of massless particles may be summarized by the equivalence of the following statements: (i) A distribution function of photons, $f(x, p)$, $p^{2}=0$, exists which is isotropic with respect to an observer $n$ and verifies the Liouville equation (1); (ii) a unit timelike vector field $n$ exists such that $\sigma=0$ and $d(a-\theta n / 3)=0$, where $\sigma, a$, and $\theta$ are the shear, acceleration, and expansion of $n$, respectively; (iii) the space-time metric is conformally stationary, that is to say, there exists a timelike vector field $\xi$ and a scalar $A$ such that $L_{\xi} g=A g$. Moreover, the observer of condition (i) [which is also the vector field of (ii)] and the conformal Killing vector of (iii) are collinear. The distribution function is an arbitrary function of the first integral associated with the conformal Killing vector, $f(x, p)=j(\xi \cdot p)$.

An important corollary for our discussion is the following: when the vector field $n$ admits a family of orthogonal surfaces, the metric satisfying condition (iii) can be written in the form

$$
d s^{2}=a^{2}\left(\eta, x^{i}\right)\left\{-\Phi^{2}\left(x^{i}\right) d \eta^{2}+\gamma_{i j}\left(x^{k}\right) d x^{i} d x^{j}\right\},
$$

where $\Phi$ and $\gamma_{i j}$ depend only on the spatial coordinates.

The observer measuring isotropic radiation will then be

$$
n=(a \Phi)^{-1} \partial_{\eta},
$$

and the temperature of the isotropic radiation will be inhomogeneous

$$
T=\frac{b}{a(\eta) \Phi\left(x^{i}\right)},
$$

$b$ being a positive constant.

One can understand this result taking into account relation (3). Let us consider a spacelike surface $\Sigma: \eta=\eta_{\Sigma}\left(x^{i}\right)$ in the past of the event $x_{0}$. A null geodesic arriving at $x_{0}$ intersects $\Sigma$ at $x_{e}=\left(\eta_{e}, x_{e}{ }^{i}\right)$, with $\eta_{e}=\eta_{\Sigma}\left(x_{e}{ }^{i}\right)$. The temperature at this point, according to (6), is $T_{e}=b /\left(a_{e} \Phi_{e}\right)$. On the other hand, the redshift of light emitted at rest with respect to $n_{e}$ and received by $n_{0}$ is given by $1+z=a_{0} \Phi_{0} /\left(a_{e} \Phi_{e}\right)$. So, taking into account relation (3) we get $T_{0}=b /\left(a_{0} \Phi_{0}\right)$ for the temperature at $x_{0}$.

\section{REVISION OF THE SACHS-WOLFE EFFECT}

The following hypotheses are appropriate to studying large angular scale anisotropies in the CMB produced by structures bigger than the horizon at decoupling time.

(1) From matter radiation decoupling up to the present, space-time may be described as a $p=0$ perturbation of an Einstein-de Sitter universe. Only the density growing mode is relevant at this epoch. Sachs and Wolfe [3] obtained this solution in comoving time-orthogonal coordinates $\left\{\bar{\eta}, \bar{x}^{i}\right\}$ :

$$
\begin{aligned}
d s^{2}=a^{2}(\bar{\eta})\left\{-d \bar{\eta}^{2}+\right. & {\left[\left[1-\frac{10}{3} \phi\right] \delta_{i j}\right.} \\
& \left.\left.-\frac{1}{3} \bar{\eta}^{2} \frac{\partial^{2} \phi}{\partial \bar{x}^{i} \partial \bar{x}^{j}}\right] d \bar{x}^{i} d \bar{x}^{j}\right\},
\end{aligned}
$$

$a(\bar{\eta})=\frac{2 \bar{\eta}^{2}}{H_{0}}$

where $\bar{\eta}$ runs from 0 to 1 at the present epoch, and $\phi$ depends on the spatial coordinates only.

(2) The Universe is inhomogeneous at scales bigger than the horizon. So density variations smaller than $10^{9}$ light years are ignored (assuming the present value of the Hubble constant to be $H_{0}=10^{-10}$ years $^{-1}$ ).

(3) Isotropic CMB with a Planck spectrum dates from the recombination epoch. The first two hypotheses are the same as in the SW paper. The third one is less restrictive because we are not assuming that decoupling occurs simultaneously for the comoving observer. Recombination did not occur instantaneously, but lasted a short period of time; however, it is represented by a last scattering surface $\Sigma: \eta=\eta_{\Sigma}\left(x^{i}\right)$. We shall use this concept but we will not identify $\Sigma$ with the surfaces $\bar{\eta}=$ const.

Let us introduce a new coordinate system $\left\{\eta, x^{i}\right\}$ by

$$
\begin{aligned}
& \bar{\eta}=\left(1+\frac{1}{3} \phi\right) \eta, \\
& \bar{x}^{i}=x^{i}+\frac{1}{6} \eta^{2} \partial_{i} \phi .
\end{aligned}
$$

Then, neglecting second-order terms in $\phi$, the metric (7) turns out to be [7]

$$
\begin{aligned}
d s^{2}=a^{2}(\eta)\{ & -\left[1+2 \phi\left(x^{i}\right)\right] d \eta^{2} \\
+ & {\left.\left[1-2 \phi\left(x^{i}\right)\right] \delta_{i j} d x^{i} d x^{j}\right\} . }
\end{aligned}
$$

The vector field of matter in these coordinates is

$$
u^{\alpha}=\left(\frac{H_{0}(1-\phi)}{2 \eta^{2}},-\frac{H_{0}}{6 \eta} \partial_{i} \phi\right) \text {. }
$$

This space-time is of the type given by (4) with $\Phi=1+\phi$, and $\gamma_{i j}=(1-2 \phi) \delta_{i j}$. On the other hand, as a consequence of hypothesis (2), scattering with electrons may be neglected and the distribution function for CMB photons will be a solution of the Liouville equation. So, if we want isotropic CMB on the last scattering surface, as is 
required in hypothesis (3), we must satisfy the conditions quoted in the previous section: i.e., (1) the observer measuring isotropic radiation must be $n=(1-\phi) a^{-1} \partial_{\eta}$, and not the comoving observer with matter $u=a^{-1} \partial_{\bar{\eta}}$, (2) the temperature on the last scattering surface $\Sigma$ will be given by

$$
T_{e}=\frac{b}{a\left(\eta_{e}\right)}\left[1-\phi\left(x_{e}{ }^{i}\right)\right]
$$

with $\eta_{e}=\eta_{\Sigma}\left(x_{e}{ }^{i}\right)$. Now, in a space-time of the type given by (9), if we have a distribution of temperature as given by (11) on a spacelike surface $\Sigma$, we will now measure (at rest with respect to observer $n_{0}$ ) a temperature $T_{0}=b\left(1-\phi_{0}\right) / a_{0}$, which is independent of the arrival direction. Therefore, if radiation was isotropic with respect to observer $n$ on the last scattering surface, it will remain isotropic in the future.

This result is independent of the shape of the last scattering surface. If we had assumed (in order to compare with SW's paper) that $\Sigma$ was a surface of constant temperature $T=T_{d}$, it would be given in comoving coordinates $\left\{\bar{\eta}, \bar{x}^{i}\right\}$ by

$$
\bar{\eta}=\sqrt{b H_{0} /\left(2 T_{d}\right)}\left[1-\frac{1}{6} \phi\left(x^{i}\right)\right] .
$$

In other words, a surface of constant temperature is not a surface $\bar{\eta}=$ const, in the coordinates used by $\mathrm{SW}$.

Finally, from (10) we conclude that matter is moving with respect to observer $n$ measuring isotropic radiation with velocity $V=V^{i} e_{i}$, with $V^{i}=-\frac{1}{3} \eta \partial_{i} \phi$ and $e_{i}$ the spacelike unit vector in the direction of $\partial_{i}$. This produces a dipolar anisotropy in the CMB measured by an observer comoving with matter. The relative velocity of matter with respect to observer $n$ may have originated during the decoupling process of matter and radiation [8].

[1] J. Bernstein, Kinetic Theory in the Expanding Universe (Cambridge University Press, Cambridge, England, 1988).

[2] P. J. E. Peebles, in 25th Anniversary of the Cosmic Background Radiation Discovery: Physical Cosmology, edited by A. Blanchard, L. Celnikier, M. Lachiez-Rey, and J. Trân Thanh Vân (Editions Frontières, Gif-sur-Yvette, France, 1991).

[3] R. K. Sachs and A. M. Wolfe, Astrophys. J. 147, 73 (1967).

[4] G. E. Tauber and J. W. Weinberg, Phys. Rev. 122, 1342 (1961).

\section{CONCLUSIONS}

We have revised the Sachs-Wolfe paper on CMB anisotropies at large angular scales. The result known as the SW effect comes from a choice of the last scattering surface $\Sigma_{d}: \bar{\eta}=$ const (defined in physical terms as a $T=$ const surface), which is not compatible with the rest of the assumptions made in the paper. The necessity of justifying this choice was recognized in SW's paper, and has recently been outlined by Stoeger et al. [9]. We have proved that under the assumptions made in SW's paper a surface of constant temperature is not a $\bar{\eta}=$ const surface [Eq. (12)].

We have proved that CMB should be isotropic at large angular scales if it was isotropic on the last scattering surface, and if the metric is given by the growing mode of a pressureless Einstein-de Sitter perturbation. This result is independent of the shape of the last scattering surface.

The conclusions would be different if we had used a perturbed Friedmann universe with a time-dependent gravitational potential $\phi$, instead of the conformal stationary metric (9). This might be necessary when considering regions of high density contrast, or in the case of a nonzero curvature background.

\section{ACKNOWLEDGMENTS}

We thank the Spanish DGICYT Project No. PB900416 for financial support. One of us (M.P.) would like to thank the Canadian Institute for Theoretical Astrophysics, where part of this paper was completed with a grant of the Conselleria de Cultura de la Generalitat Valenciana.

[5] J. Ehlers, P. Geren, and R. K. Sachs, J. Math. Phys. 9, 1344 (1968).

[6] J. J. Ferrando, J. A. Morales, and M. Portilla, Phys. Rev. D 46, 578 (1992).

[7] J. Moreno, Ph.D. thesis, Universidad de Valencia, 1992.

[8] J. J. Ferrando, J. A. Morales, and M. Portilla, in Proceedings of the "Journees Rélativistes" [Class. Quantum Grav. (to be published)].

[9] W. R. Stoeger, G. F. R. Ellis, and B. G. Schmidt, Gen. Relativ. Gravit. 23, 1169 (1992). 